\title{
A call for accurate phenotype definition in the study of complex disorders
}

\section{To the editor:}

Gretarsdottir et al. recently reported on the association between risk of ischemic stroke and the gene encoding phosphodiesterase 4D $(P D E 4 D)^{1}$. There was no significant association with ischemic stroke as a whole ${ }^{1}$, and so the authors focused on various etiological subgroups of stroke. They found that different haplotypes were associated with different risk of combined carotid and cardiogenic strokes. Their work warrants serious criticism. There is an important methodological problem relating to combining individuals with cardiogenic and carotid stroke into one subgroup for genetic analysis. Combined carotid and cardiogenic strokes represented $40-45 \%$ of all ischemic strokes in the authors' sample ${ }^{1,2}$, which means that they excluded $55-60 \%$ of individuals with stroke from the genetic association study. Excluding that many individuals (and losing that much genetic information) is appropriate only if it refines the stroke phenotype in the remaining sample. Unfortunately, this was not the case in the study of Gretarsdottir et al.

The authors claim that cardiogenic and carotid strokes are most clearly related to atherosclerosis. This is true for carotid strokes (14-16\% of all ischemic strokes in their population ${ }^{1,2}$ ) but not for cardiogenic strokes (24-29\% of total). Data from the literature show that only $25 \%$ of cardiogenic strokes directly result from complications of coronary atherosclerosis ${ }^{3}$, whereas $\sim 65 \%$ are due to valvular heart disease or atrial fibrillation (with less than $20 \%$ of all atrial fibrillations being attributable to coronary heart disease $\left.{ }^{4}\right)$. The combined subgroup resulting from the admixture of carotid and cardiogenic strokes is, in fact, highly heterogeneous and, in our opinion, unsuitable for genetic analysis. Readers should receive with caution the authors' assumptions regarding the putative role of $P D E 4 D$ in atherogenesis and subsequent risk of stroke. In agreement with this caveat, we note that PDE4D genotypes or haplotypes do not influence the risk of peripheral artery occlusive disease or coronary artery disease (see Supplementary Table 7 from ref. 1), although atherosclerosis is the cause of both disorders in more than $90 \%$ of cases.

The genetic analysis of the whole group of individuals with ischemic stroke showed a possible though nonsignificant association of anonymous markers at the PDE4D locus with stroke. In this context, the additional finding of downregulation of several PDE $4 D$ mRNA isoforms in individuals with ischemic stroke further suggests that $P D E 4 D$ is a very good candidate for genetic modulation of ischemic stroke risk. Gretarsdottir et al. only considered the possible involvement of $P D E 4 D$ in atherogenesis, which is supported by few experimental data ${ }^{1}$. By contrast, phosphodiesterase 4 has repeatedly been reported to be involved in susceptibility to ischemic brain damage in animal models ${ }^{5-8}$. Such a mechanism directly related to cerebral ischemia could explain why the PDE4D locus was found to be in linkage with stroke regardless of cause in the Icelandic population $^{2}$.

It seems that the PDE4D functional variant that influences the risk of ischemic stroke has yet to be identified, as statistically significant associations were found only in a pathophysiologically inadequate subgroup of individuals in the present study. Genotyping studies are becoming more comprehensive and including growing numbers of polymorphisms in each study. Accurate phenotype definition seems more than ever to be a necessary prerequisite to establish reliable genotype-phenotype relationships in the study of complex disorders.

\section{Benoît Funalot ${ }^{1,2}$, Olivier Varenne ${ }^{3}$} \& Jean-Louis Mas ${ }^{1}$

${ }^{1}$ Department of Neurology, Hôpital Sainte-Anne, 1 rue Cabanis, 75014 Paris, France. ${ }^{2}$ Institut

National de la Santé et de la Recherche Médicale,
Unité 573, Centre Paul-Broca, Paris, France. ${ }^{3}$ Department of Cardiology, Hôpital Cochin, Paris, France. Correspondence should be addressed to B.F. (benoit.funalot@broca.inserm.fr).

1. Gretarsdottir, S. et al. Nat. Genet. 35, 131-138 (2003).

2. Gretarsdottir, S. et al. Am. J. Hum. Genet. 70, 593-603 (2002).

3. Cerebral Embolism Task Force. Arch. Neurol. 46, 727-743 (1989).

4. Levy, S. et al. Circulation 99, 3028-3035 (1999).

5. Nagasawa, H., Araki, T. \& Kogure, K. Neurosci. Lett. 144, 34-37 (1992)

6. Kato, H., Araki, T., Itoyama, Y. \& Kogure, K. Eur. J. Pharmacol. 272, 107-110 (1995).

7. Block, F., Tondar, A., Schmidt, W. \& Schwarz, M. Neuroreport 8, 3829-3832 (1997).

8. Nagakura, A., Niimura, M. \& Takeo, S. Br. J. Pharmacol. 135, 178-1793 (2002).

\section{In reply}

Definitions of clinical phenotypes are often arrived at through consensus criteria that may have little to do with the underlying biology. Stroke is an entity with subtypes that lack clear biological correlates.

Funalot et al. argue that there is a methodological problem in our genetic association study, namely, our combined analyses of the carotid and cardiogenic stroke subtypes. They do not agree that atherosclerosis is the primary pathological process for both carotid and cardiogenic stroke and claim that only $25 \%$ of cardiogenic strokes result directly from coronary atherosclerosis and $65 \%$ are due to valvular heart disease or atrial fibrillation $(<20 \%$ of those being attributable to coronary atherosclerosis).

This is a different pattern from that seen in our Icelandic stroke cohort, in which at least $47 \%$ of individuals with cardiogenic stroke have coronary artery disease. Atrial fibrillation is observed in $79 \%$ of individuals with cardiogenic stroke, and $44 \%$ of those have documented coronary artery disease. Recent studies, especially in Scandinavian populations, of atrial fibrillation show that 